\title{
Análisis geomático del cambio de uso del suelo en la subcuenca río Copalita, Oaxaca
}

\section{Geomatic analysis of land use change in the Copalita River sub-basin, Oaxaca}

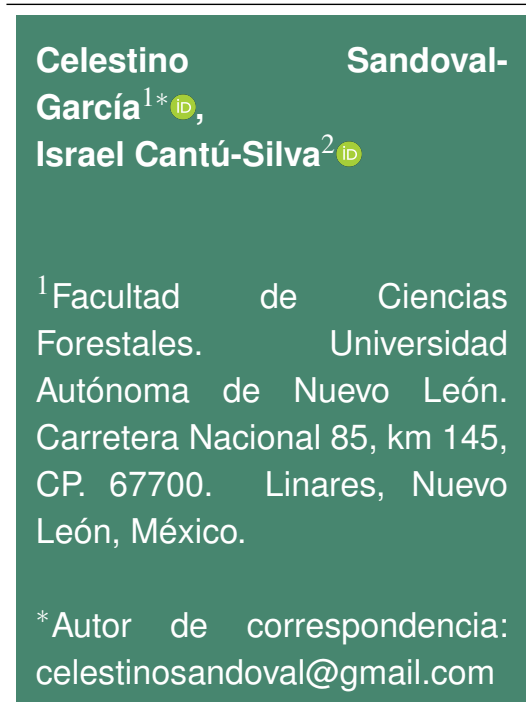

Artículo científico

Recibido: 27 de febrero 2021

Aceptado: 19 de octubre 2021

Como citar: Sandoval-

García C, Cantú-Silva

(2021) Análisis geomático

del cambio de uso del suelo

en la subcuenca río Co-

palita, Oaxaca. Ecosistemas

y Recursos Agropecuarios

Núm. Esp. II: e2915. DOI:

10.19136/era.a8nll.2915
RESUMEN. El presente estudio tiene como objetivo evaluar el cambio de cobertura de vegetación y uso del suelo de la subcuenca Río Copalita, Oaxaca, para el periodo 1995-2015. Para determinar el cambio de uso del suelo se utilizaron ortofotos para el periodo 1995 e imágenes satelitales de alta resolución para el año 2015, mismos que fueron procesados y analizados en el programa QGIS 2.18.25. Los resultados indican que selva mediana caducifolia presentó una tasa de cambio de $-0.84 \%$, sabana $-0.66 \%$, bosque de pino-encino $-0.65 \%$, selva mediana subperennifolia $-0.52 \%$, bosque mesófilo de montaña $-0.27 \%$, selva mediana subcaducifolia $-0.23 \%$, bosque de encino-pino $-0.23 \%$, bosque de galería $-0.17 \%$ y bosque de oyamel con $-0.05 \%$. El bosque de pino presentó una ganancia de $0.35 \%$, agricultura $0.66 \%$ y pastizal $1.12 \%$. Dichos cambios se dan en toda la subcuenca, sin embargo, en la parte alta principalmente se observó una recuperación de la cobertura forestal, ya que los sistemas de producción agrícolas y pastizales se convirtieron en plantaciones y reforestaciones de pino. Por lo que es importante seguir impulsando diferentes prácticas que permitan la conservación y recuperación de áreas degradadas de la subcuenca, tales como plantaciones de pino, sistemas agrosilvícolas, manejo de acahuales y pago de servicios ambientales hidrológicos.

Palabras clave: Bosque de pino, cambio de la cobertura del suelo, imágenes satelitales, QGIS, sistema de usos del suelo.

ABSTRACT. The present study aims to evaluate the change in vegetation cover and land use of the Copalita River sub-basin, Oaxaca, for the period 19952015. To determine the land use change, orthophotos were used for the 1995 period and high-resolution satellite images for 2015 , which were processed and analyzed in the QGIS 2.18.25 program. The results indicate that medium deciduous forest presented a change rate of $-0.84 \%$, savannah $-0.66 \%$, pine-oak forest $-0.65 \%$, medium sub-evergreen forest $-0.52 \%$, mountain mesophilic forest $-0.27 \%$, medium subdeciduous forest $-0.23 \%$, oak-pine forest $-0.23 \%$, gallery forest $-0.17 \%$ and oyamel forest with $-0.05 \%$. Pine forest presented a gain of $0.35 \%$, agriculture $0.66 \%$ and pasture $1.12 \%$. These changes occur throughout the sub-basin, however, in the upper part, mainly a recovery of forest cover was observed, since agricultural production systems and pastures were converted into plantations and reforestations. Therefore, it is important to continue promoting different practices that allow the conservation and recovery of degraded areas of the sub-basin, such as pine plantations, agrosilvicultural systems, management of fragmented forest and paying for the hydrological services.

Key words: Pine forest, change of land cover, satellite images, QGIS, land use systems. 


\section{INTRODUCCIÓN}

Durante el periodo de 1972 y 1982 en varios estados del país operó el Programa Nacional de Desmonte (PRONADE), siendo la Sierra Sur de Oaxaca una de las regiones donde se implementó este programa. En ese entonces los ejidatarios y comuneros abrieron nuevas áreas para la agricultura principalmente en áreas forestales (Moreno-Unda et al. 2019). Sin embargo, el cambio de uso del suelo continúa, ya que el estado de Oaxaca presentó una deforestación promedio de 18757 ha para el periodo de 2001 al 2018, siendo las áreas forestales las más afectadas (CONAFOR 2020). El cambio de uso del suelo se mantiene, debido a que se requieren nuevos espacios para la agricultura, la ganadería, así como para el asentamiento humano ante el incremento de la población, la cual se registra en 126014024 personas en México para el año 2020 (INEGI 2020).

Ante este crecimiento, existe una mayor demanda de bienes y servicios para satisfacer las necesidades de la población, por lo que existe una presión hacia los diferentes recursos naturales, siendo el forestal uno de los más afectados, y por consecuencia el suelo (Palacio-Prieto et al. 2015). El cambio de uso de suelo es uno de los problemas del ambiente más importantes debido a las consecuencias que conlleva, tales como el cambio en el micro y mesoclima; dependiendo de la superficie en el que se da el cambio de uso, conlleva la liberación de $\mathrm{CO}_{2}$ al medio, la disminución de la infiltración de agua al suelo, el incremento de la escorrentía y la pérdida de la biodiversidad y del hábitat, así como de los servicios ambientales (Ihl y Bautista-Zuñiga 2019). Lo anterior, ha provocado que el cambio de uso del suelo, especialmente el cambio de vegetación natural en agricultura y usos urbanos, pueda modificar directamente el ciclo hidrológico, lo cual resulta en un aumento de las inundaciones y una disminución en la recarga de aguas subterráneas, entre otros efectos (Brauman et al. 2014, Sandoval y Tiburan 2019).

En este sentido, dada la importancia de la subcuenca Río Copalita en la región sur de Oaxaca, es importante cuantificar los cambios de cobertura de vegetación y uso de suelo. Pero dada las condiciones topográficas y lo poco accesible en algunas áreas, es necesario el uso de nuevas tecnologías como la teledetección, herramienta usada para la evaluación de los recursos naturales y que permite establecer indicadores de degradación y conservación, y de manera particular la dinámica del cambio de uso del suelo y cobertura en una determinada región. La teledetección espacial permite seguir procesos dinámicos, ya que las imágenes son captadas por un sensor a la Tierra desde la órbita de manera estable y repetida (Chuvieco 2002). Así mismo, las variaciones temporales y espaciales de coberturas son un importante indicador de las dinámicas de los ecosistemas (Gonzaga-Aguilar 2015). La Sierra Sur y Costa del estado de Oaxaca, son dos regiones que albergan una gran biodiversidad en los diferentes ecosistemas, además de los bienes y servicios que ofrecen a la sociedad. Pero en los últimos años ha existido una fuerte presión por las diferentes actividades antropogénicas provocando un deterioro ambiental. Lo anterior, permite valorar el uso de imágenes de alta resolución en la plataforma SASPlanet de libre acceso, las cuales están disponibles hasta el año 2015, considerando así un periodo de evaluación de 20 años. Con base en lo anterior, el objetivo del presente estudio fue realizar un análisis geomático del cambio de uso de suelo en la subcuenca Río Copalita, Oaxaca, México, para el periodo 1995-2015.

\section{MATERIALES Y MÉTODOS}

\section{Ubicación del área de estudio}

La subcuenca Río Copalita se encuentra en la Región Hidrológica 21, que corresponde a la región V, Pacífico Sur, incluyendo región de la Sierra Sur y Costa del estado de Oaxaca, con una superficie de 166252.40 ha (Figura 1). Los tipos de clima que presenta son Cálido subhúmedo, Semicálido húmedo, Semifrío subhúmedo y Templado subhúmedo (García 2004). La precipitación pluvial varía de 900 a 2 $200 \mathrm{~mm}$ al año y la temperatura fluctúa de 15 a $26^{\circ} \mathrm{C}$ (INEGI 2004). Los suelos más representativos que presenta son Cambisol, Fluvisol, Luvisol, Leptosol, Fhaeozem, Regosol y Umbrisol (INEGI 2014a, IUSSWRB-FAO 2015). Los tipos de vegetación más impor- 


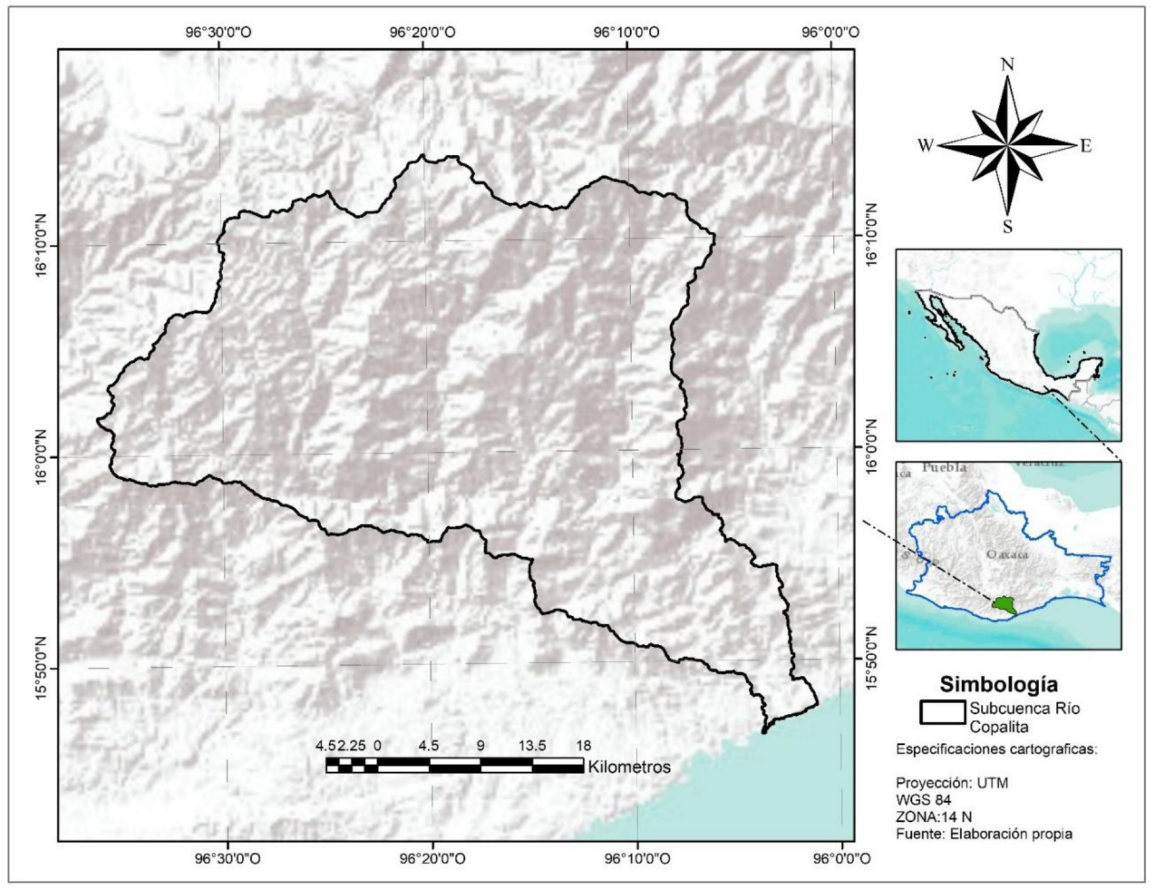

Figura 1. Ubicación de la subcuenca Río Copalita, Oaxaca.

tantes en la subcuenca son bosque de pino, bosque de pino-encino, bosque de oyamel, bosque mesófilo de montaña, selva mediana perennifolia, sabana, bosque de galería, selva mediana caducifolia, mismos que en su mayoría se consideran es vegetación primaria y en menor superficie, secundaria (Tabla 1) (INEGI 2014b, INEGI 2017).

\section{Proceso de selección de ortofotos e imágenes satelitales}

Para determinar el cambio de cobertura y uso del suelo, se obtuvieron las siguientes ortofotos del INEGI: D14B18a, D14B18b, D14B18c, D14B18d, D14B18e, D14B18f, D14B19a, D14B19b, D14B19c, D14B19d, D14B19e, D14B19f, E14D88a, E14D88b, E14D88c, E14D88d, E14D88e, E14D88f, E14D89a, E14D89b, E14D89c, E14D89d, E14D89e, E14D89f a una escala 1:75 000 para el año 1995. Para 2015, se realizó la descarga de imágenes de alta resolución a una escala 1:4 000 del programa SASPlanet (2018).

\section{Clasificación de imágenes}

La clasificación de las ortofotos e imágenes satelitales obtenidas se usó el procedimiento KMC (K-Means Cluster Analysis), un método que permite hacer una clasificación no supervisada y agrupación en clúster para analizar características de las ortofotos e imágenes satelitales. En las ortofotos este método se utilizó para agrupar las clases espectrales (Pontius et al. 2004, Gonzaga-Aguilar 2015). La clasificación supervisada se realizó mediante sitios de entrenamiento a partir de la información que se obtuvo en campo, mediante sitios de muestreo de $500 \mathrm{~m}^{2}$ en los diferentes ecosistemas para asegurar que la clasificación fuese la correcta. Así mismo, se realizó la ejecución de 16 clases de manera automatizada. Las cuales se resumieron en 12 clases de cobertura y uso del suelo. Todos los análisis y procedimientos se realizaron en el sistema de información geográfica QGIS 2.18.25 (QGIS Development Team 2019). De acuerdo con Pauleit et al. (2005), el uso del suelo se define en función de las actividades económicas, y la cobertura representa a los elementos que cubren el suelo, como son los diferentes tipos de vegetación que existen. Para realizar el reconocimiento de los diferentes usos del suelo y cobertura del área de es- 
Tabla 1. Principales especies de los ecosistemas más representativas de la subcuenca Río Copalita, Oaxaca.

\begin{tabular}{|c|c|}
\hline Ecosistema & Principales especies \\
\hline Bosque de pino & $\begin{array}{l}\text { Pinus oaxacana Mirov., P. patula Schl. et Cham, P. pseudostrobus Lindl., P. teocote Schiede ex Schltdl., P. oocarpa } \\
\text { Schiede ex Schltdl., P. douglasiana Martínez, P. leiophyilla Schl. et Cham., P. ayacahuite Ehren., P. rudis Endl., P. } \\
\text { maximinoi H. E. Moore. }\end{array}$ \\
\hline Bosque de pino-encino & $\begin{array}{l}\text { Pinus oaxacana Mirov., P. patula Schl. et Cham, P. pseudostrobus Lindl., P. teocote Schiede ex Schltdl., P. oocarpa } \\
\text { Schiede ex Schltdl., P. douglasina Martínez, P. leiophyilla Schl. et Cham., P. ayacahuite Ehren., P. rudis Endl., P. } \\
\text { maximinoi H. E. Moore. Quercus laurina Bonpl., Q. crassifolia Humb. \& Bonpl., Q. magnolifolia Née, Q. elliptica Née, } \\
\text { Q. resinosa Liebm., Q. candicans Née, Q. greggii A.DC., Quercus rugosa Née }\end{array}$ \\
\hline $\begin{array}{l}\text { Selva mediana caducifolia } \\
\text { y selva mediana subca- } \\
\text { ducifolia }\end{array}$ & $\begin{array}{l}\text { Manilkara zapota (L.) P. van Royen., Bursera simaruba (L.) Sarg., Ficus goldmanii Standl, Cordia alliodora (Ruiz } \\
\text { \& Pav.) Oken., Tabebuia rosea (Bertol.) A. DC., Cecropia obtusifolia Bertol., Ceiba pentandra (L.) Gaertn., En- } \\
\text { terolobium cyclocarpum (Jacq.) Griseb., Hevea brasiliensis Muell. Arg., Ouratea guatemalensis Engl., Chamaedora } \\
\text { elegans Mat., y C. tepejilote Liebmn. }\end{array}$ \\
\hline $\begin{array}{l}\text { Selva mediana subperen- } \\
\text { nifolia }\end{array}$ & $\begin{array}{l}\text { Spondias mombium L., Stemmadenia donnell-smithii (Rose) Woodson., Calophyllum brasiliense Cambess., Croton } \\
\text { reflexifolius Kunth., Acacia cornigera (L.) Willd., Gliricidia sepium (Jacq.) Steud., Inga jinicuil G. Don., Lysiloma aca- } \\
\text { pulcensis (Kunth) Benth., Pithecellobium dulce (Roxb.) Benth., Pithecellobium lanceolatum (Humb. y Bonpl. ex } \\
\text { Willd.) Benth., Conostegia xalapensis (Bonpl.) D. Don ex DC., Brosimum alicastrum Sw., Guazuma ulmifolia Lam. }\end{array}$ \\
\hline $\begin{array}{l}\text { Bosque mesófilo de mon- } \\
\text { taña }\end{array}$ & $\begin{array}{l}\text { Cornus disciflora DC., Quercus ocoteifolia Liebm., Psychotria galeottiana M. Martens., Cleyera theaeoides (Sw.) } \\
\text { Choisy., Oreopanax xalapensis (Kunth) Decne., Ternstroemia tepezapote Schltdl. et Cham., Chiranthodendron pen- } \\
\text { tadactylon Larreat., Rapanea juergensenii Mez., Clethra mexicana DC., Styrax argenteus C. Presl., Vallesia auran- } \\
\text { tiaca (M. Martens et Galeotti) J. F. Morales., Turpinia occidentalis (Sw.) G.Don., Quercus skinneri Benth., Pinus } \\
\text { chiapensis Martínez Andresen., Berberis moranensis Schult. et Schult.f. }\end{array}$ \\
\hline Bosque de oyamel & Abies religiosa (Kunth) Schltdl. \& Cham. \\
\hline Sabana & Crescentia cujete L., Sabal mexicana Mart., Byrsonima crassifolia (L.) Kunth \\
\hline Bosque de galería & Laguncularia racemosa (L.) C.F., Salix humboldtiana Willd. \\
\hline
\end{tabular}

Fuente: Perry, 1991, Mejía-Domínguez et al. 2004, Pennington y Sarukhán 2005, Salas-Morales et al. 2007.

tudio, se generó una tipología que se puede observar en la Tabla 2.

\section{Determinación de pérdidas y ganancias de tipo de cobertura}

Para determinar la pérdida y ganancia de cobertura se verificaron las etiquetas y se realizó la corrección de los polígonos generados en el programa QGIS 2.18.25. De esta manera se pudo obtener la tendencia de cambio de cobertura y uso de suelo, mediante la sobreposición y posteriormente, calcular las diferencias entre los cortes de tiempo analizados. La determinación de la pérdida o ganancia de cobertura de los diferentes ecosistemas se obtuvo mediante la construcción de una matriz de transición y una de tasa de cambio para los dos periodos. Para determinar la tasa de cambio, se utilizó la fórmula adaptada por Palacio-Prieto et al. (2004).

$$
\delta_{n}\left[\left(\frac{s_{2}}{s_{1}}\right)^{1 / n}-1\right] \times 100
$$

Donde: $\delta_{n}=$ Tasa de cambio expresada en porcentaje, $s_{1}=$ Superficie de la fecha $1, s_{2}=$ Superficie de la fecha 2, $n=$ Número de años entre las dos fechas.

\section{RESULTADOS}

\section{Cambios en el uso de suelo y vegetación}

Después de realizar la clasificación supervisada de las imágenes de alta resolución, se lograron identificar 16 tipos de uso de suelo y vegetación. Las clases de ríos, caminos, líneas de luz y asentamientos humanos no mostraron cambios significativos, por lo que en la clasificación solo se consideraron 12 tipos. En el Tabla 3, se presenta la superficie que ocupa cada tipo de uso del suelo, así como la tasa de cambio de uso en la subcuenca para el periodo 1995-2015.

En el año 1995 se observó una fuerte presión en los bosques de pino, bosque mesófilo de montaña y bosque de pino-encino, como consecuencia del cambio de uso del suelo que se practica con el sistema de roza-tumba-quema con fines agrícolas y ganaderos, así como la tala clandestina, principalmente en la parte alta de la subcuenca, lo cual propició una disminución en la cobertura vegetal. En 2015, en la mayoría de los usos del suelo se presentó una disminución en la superficie, excepto bosque de pino, pastizal y agricultura (Tabla 3 ). 


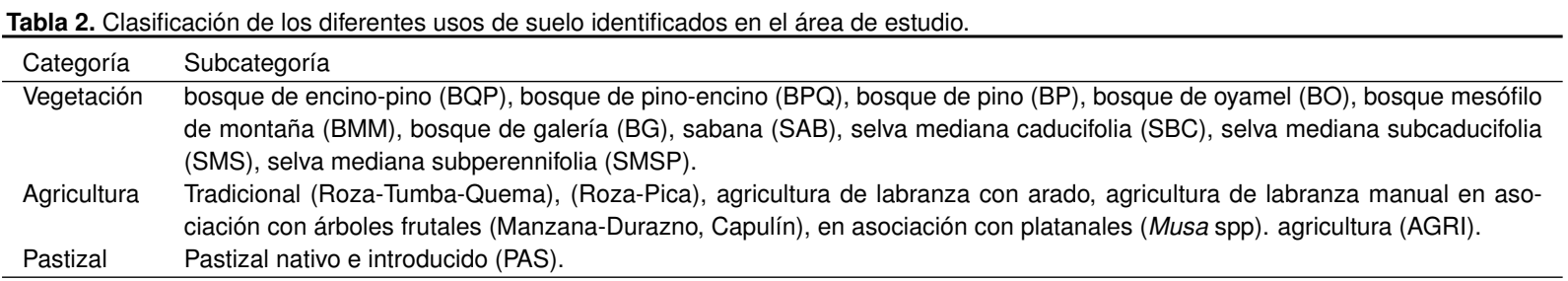

Tabla 3. Superficie (ha) y tasa de cambio anual (\%) para las diferentes coberturas vegetales y usos del suelo.

\begin{tabular}{lrrrr}
\hline & & & Diferencia & TC \\
Cobertura de vegetación y uso de suelo & 1995 & 2015 & $1995-2015$ & Anual \\
\cline { 2 - 5 } & ha & ha & ha & $\%$ \\
\hline Bosque de encino-pino & 1552.48 & 1483.79 & -68.69 & -0.23 \\
Bosque de pino-encino & 25513.98 & 22414.14 & -3099.84 & -0.65 \\
Bosque de pino & 46735.55 & 50110.92 & 3375.37 & 0.35 \\
Bosque de oyamel & 877.44 & 869.30 & -8.14 & -0.05 \\
Bosque mesófilo de montaña & 26670.26 & 25256.76 & -1413.5 & -0.27 \\
Bosque de galería & 375.85 & 362.94 & -12.91 & -0.17 \\
Sabana & 1321.79 & 1157.03 & -164.76 & -0.66 \\
Selva mediana caducifolia & 11665.71 & 9861.17 & -1804.54 & -0.84 \\
Selva mediana subcaducifolia & 5296.68 & 5056.16 & -240.52 & -0.23 \\
Selva mediana subperennifolia & 10376.01 & 9356.73 & -1019.28 & -0.52 \\
Pastizal & 7224.01 & 9024.17 & 1800.16 & 1.12 \\
Agricultura & 18910.66 & 21567.34 & 2656.68 & 0.66 \\
\hline TC $=$ Tasa de cambio & & & &
\end{tabular}

El cambio de cobertura y uso del suelo resultó ser un proceso muy dinámico en la subcuenca Río Copalita de 1995 hasta 2015. Las clases de vegetación más vulnerables por la disminución de superficie fueron: selva mediana subperennifolia, sabana, bosque de pino-encino y bosque mesófilo de montaña. Los cambios de uso del suelo más comunes que se observaron fueron: de forestal a agricultura, agricultura a pastizal, agricultura a plantación o reforestación de pino y de pastizal a forestal. Con base en el análisis y de acuerdo con la clasificación de uso del suelo y vegetación para 1995 se pueden observar las áreas de agricultura predominando en la parte alta de la subcuenca (Figura 2), mientras que en 2015 se observa una disminución de los sistemas agrícolas en la parte noroeste en la misma zona (Figura 3).

\section{Estimación del cambio de uso de suelo}

En el periodo 1995-2015 de los usos de suelo y tipos de vegetación que existen en la subcuenca, se observó un incremento para bosque de pino (BP) de 3375.37 ha, agricultura (AGRI) de 2665.68 ha y pastizal (PAS) de 1800.16 ha, con respecto a su superficie original. El incremento de BP se debió a las reforestaciones y plantaciones forestales establecidas con especies nativas de la región, mientras que las siguientes coberturas de vegetación presentaron una disminución: bosque de pino-encino (BPQ) de 3099.8 ha, selva mediana caducifolia (SMC) de 1 804.5 ha, bosque mesófilo de montaña (BMM) de 1 400 ha, selva mediana subperennifolia (SMSP) de 1019.3 ha, selva mediana subcaducifolia (SMS) de 240.52 ha, sabana (SAB) de 164.76 ha, bosque de galería (BG) de 12.91 ha y bosque de oyamel (BO) de 8.14 ha (Figura 4).

Por otra parte, en la Figura 5 se observa la tasa de cambio de uso del suelo que presentó la subcuenca Río Copalita, Oaxaca, donde el bosque de pino mostró una ganancia de $0.35 \%$, así como el uso agrícola $(0.66 \%)$ y pastizal $(1.12 \%)$, mientras que el resto de los usos del suelo presentaron una pérdida durante el periodo de 20 años. 


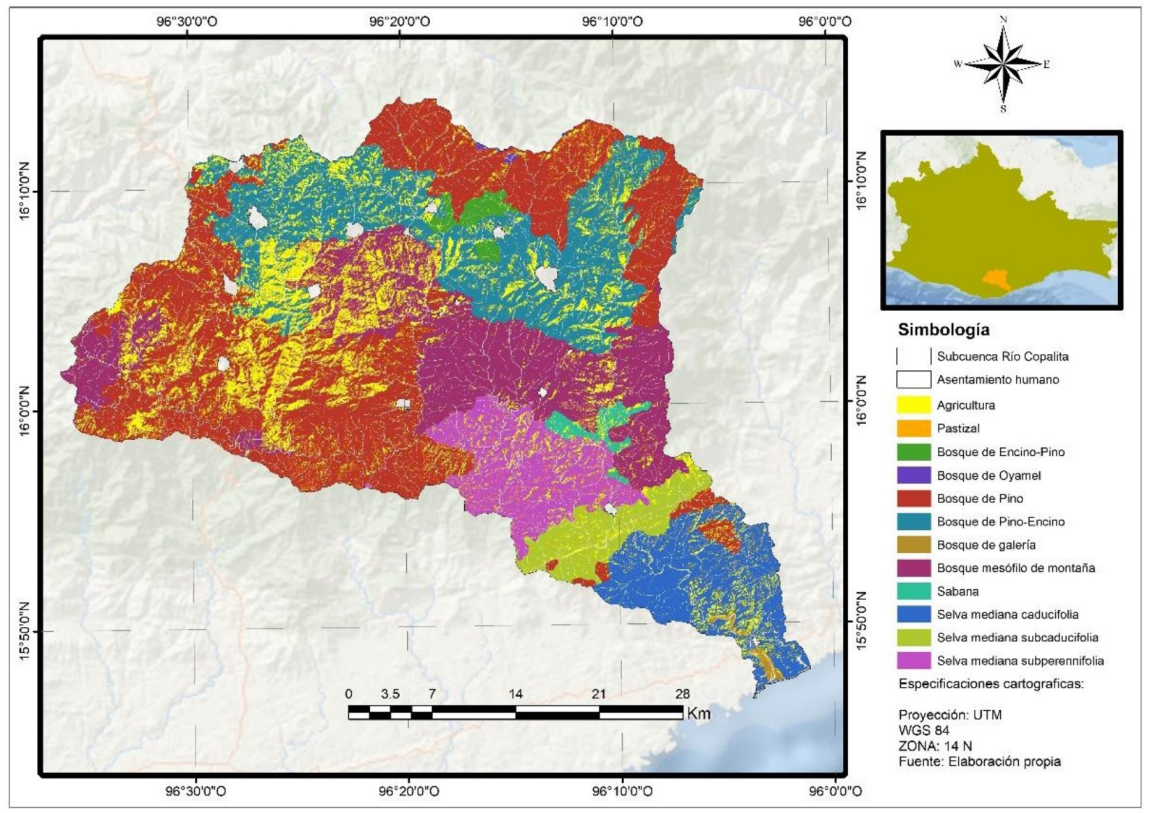

Figura 2. Clasificación de la cobertura de la vegetación y tipos de uso de suelo del año 1995, subcuenca Río Copalita.

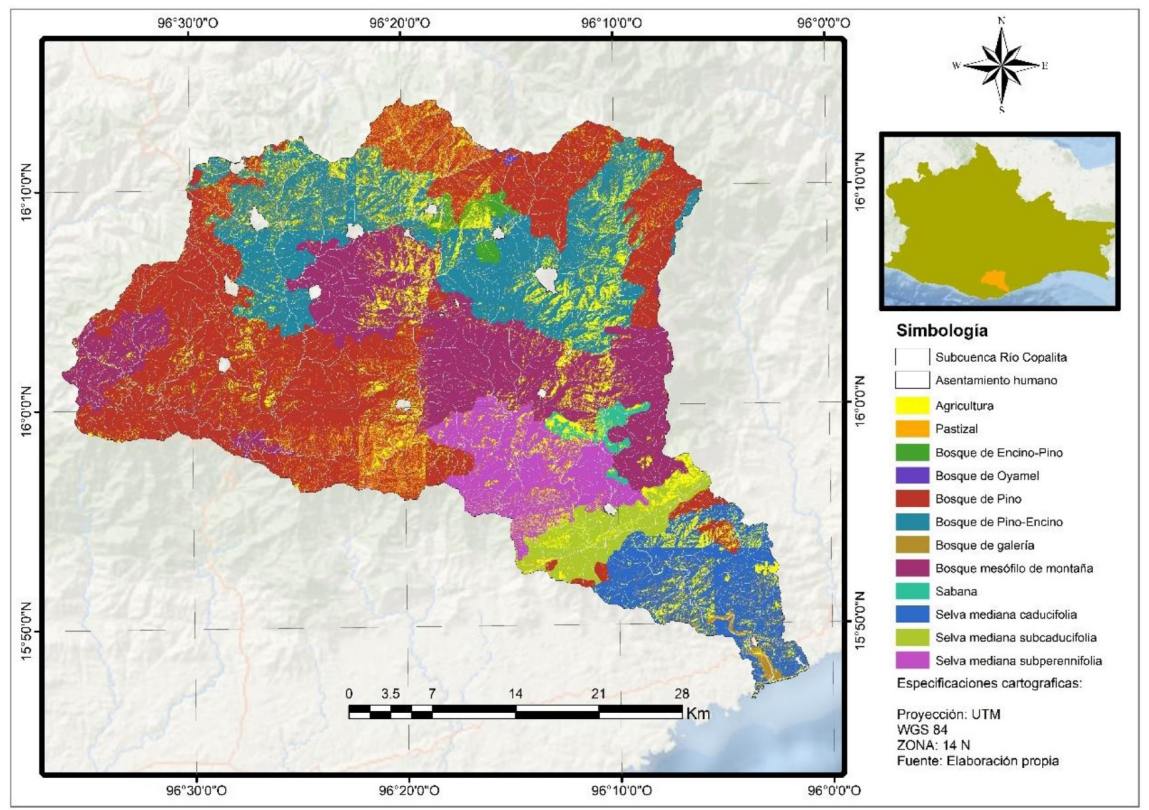

Figura 3. Clasificación de la cobertura de la vegetación y tipos de uso de suelo del año 2015, subcuenca Río Copalita.

\section{DISCUSIÓN}

\section{Cambios en el uso de suelo y vegetación}

La subcuenca Río Copalita presentó un cam- bio de usos del suelo muy dinámico durante el periodo evaluado. El mayor cambio hasta 1995 ocurrió en la parte alta y media de la subcuenca, a raíz del Programa Nacional de Desmonte (PRONADE) imple- 


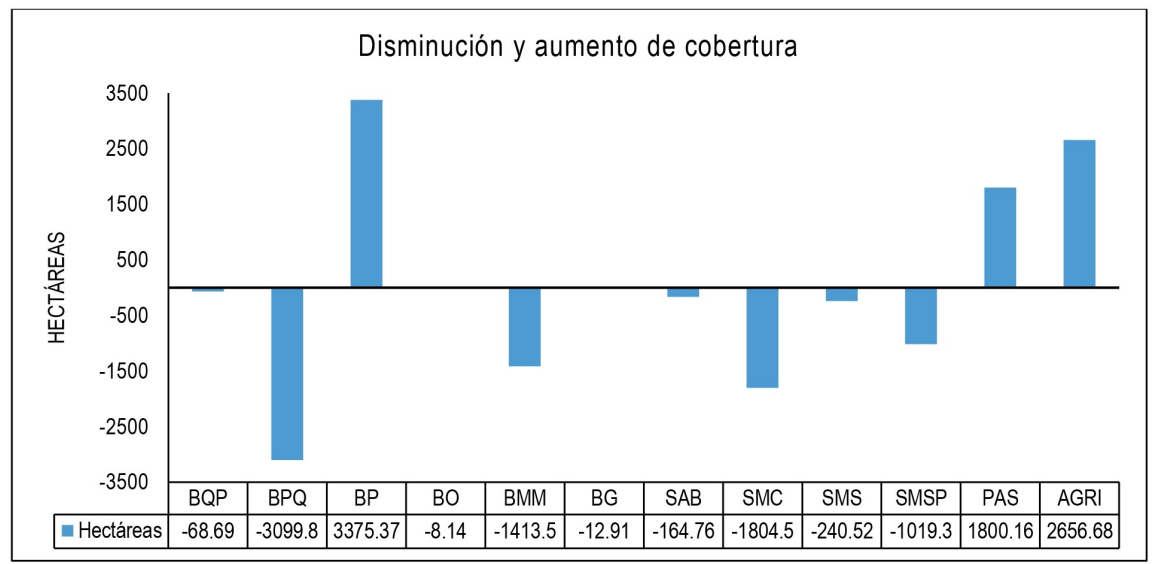

Figura 4. Disminución o aumento de la cobertura de vegetación y uso del suelo de la subcuenca Río Copalita, Oaxaca, durante el periodo 1995-2015. BQP = bosque de encino-pino, $\mathrm{BPQ}=$ bosque de pino-encino, $\mathrm{BP}=$ bosque de pino, $\mathrm{BO}=$ bosque de oyamel, $\mathrm{BMM}=$ bosque mesófilo de montaña, $B G=$ bosque de galería, $\mathrm{SAB}=$ sabana, $\mathrm{SMC}$ = selva mediana caducifolia, $\mathrm{SMS}$ = selva mediana subcaducifolia, $\mathrm{SMSP}=$ selva mediana subperennifolia, $\mathrm{PAS}=$ pastizal, $\mathrm{AGRI}=$ agricultura

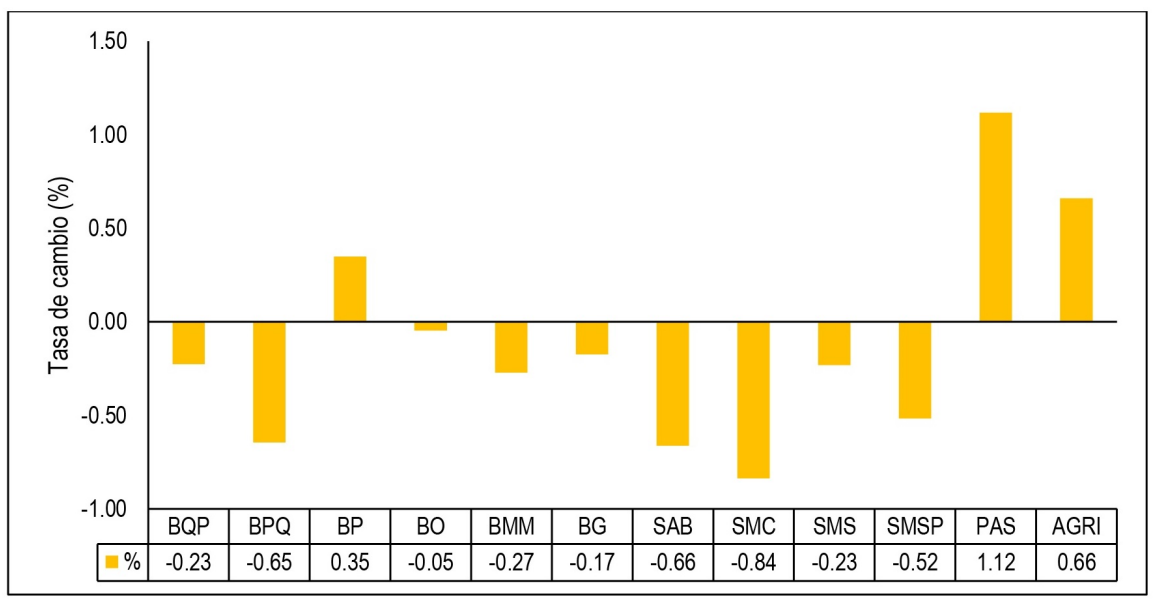

Figura 5. Tasa de cambio de cobertura de vegetación y uso del suelo de la subcuenca Río Copalita, Oaxaca, durante el periodo 1995-2015.

mentado en la Sierra Sur de Oaxaca, el cual permitió a los campesinos convertir nuevas áreas para la agricultura dentro de los bosques (Moreno-Unda et al. 2019).

Con base en la evaluación, se pudo constatar que la mayoría de los usos de suelo y coberturas presentaron una pérdida, pero tuvo una tasa positiva en cuanto a la recuperación de superficie del bosque de pino de 3375.37 ha, debido a las actividades de reforestación y al establecimiento de plantaciones de pino a pequeña escala, usando diferentes especies nativas de la región como Pinus oaxacana Mirov., $P$. ayacahuite Ehrenb. Ex Schltdl., P. douglasiana
Martínez, P. patula var. longepedunculata Loock ex Martínez, y P. leiophylla Schl. \& Cham. (CONAFOR 2016).

Con la implementación de los diferentes programas de gobierno como el Programa Nacional de Reforestación (PRONARE), Programa de Plantaciones Forestales Comerciales (PRODEPLAN) (SEMARNAP 2000) y ProÁrbol, se ha favorecido la recuperación de áreas que anteriormente se dedicaban a la agricultura y ganadería, las cuales actualmente cuentan con vegetación arbórea (CONAFOR 2016). Por otra parte, la presencia de diferentes organizaciones ha favorecido la implementación de 
varios proyectos para la restauración y conservación de áreas prioritarias, como la Fundación Gonzalo Río Arronte, que en el 2004 financió diferentes proyectos socioambientales con los que se logró la reforestación de 2625 ha de diferentes especies nativas de la región, principalmente en la parte alta y media de la subcuenca Copalita-Zimatán-Huatulco (Mansourian et al. 2020).

Pero en la parte baja de la subcuenca los cambios de uso del suelo fueron propiciados en muchos casos por los diferentes programas de gobierno como PROCAMPO y PROGAN, en los que se promovía la remoción de la vegetación natural para el establecimiento de agricultura y ganadería (Ellis et al. 2016). En 2015 la superficie de la mayoría de la vegetación se redujo; los tipos de vegetación que presentaron una mayor disminución fueron la selva mediana caducifolia con $15.46 \%$, bosque de pinoencino con $12.15 \%$ y sabana con $12.46 \%$. Esta pérdida de cobertura ocurrió debido al establecimiento de nuevas áreas para la agricultura y la ganadería. Lo anterior, coincide con lo reportado por Leija-Loredo et al. (2016) quienes realizaron un estudio en dos municipios de la costa de Oaxaca, donde mencionan que de continuar con la tendencia de cambio de uso de suelo, para el 2025 se habrán perdido 37937 ha de selvas, 650 ha de bosque y 887 ha de manglares, ecosistemas considerados de gran importancia para la conservación y protección de la biodiversidad. Los resultados obtenidos coinciden con lo señalado por Escandón-Calderón et al. (2018) en su estudio realizado en el estado de Morelos, donde encontraron que los usos de suelo que tuvieron mayor transformación fueron los bosques y selvas bajas, convirtiéndose a usos agrícolas y pastizales para el periodo evaluado; así mismo, mencionan que la dinámica del cambio de uso es compleja, debido a las condiciones del lugar. Por otra parte, Camacho-Sanabria et al. (2015) en la zona suroeste del Estado de México encontraron que en un periodo de 10 años, la cobertura de bosque disminuyó considerablemente, siendo el uso agropecuario el que presentó la mayor superficie de cambio con 19113 ha, y en menor cantidad las áreas de asentamiento humano y agricultura tecnificada. Así mismo, Nené-Preciado et al.
(2017) evaluaron dos cuencas costeras de Jalisco, encontrando una pérdida de 4000 ha para bosques tropicales en la cuenca Arroyo Seco y 7100 ha para la cuenca María García, donde los usos agropecuarios aumentaron en cada cuenca en un $55 \%$ y $175 \%$, respectivamente. Lo anterior, concuerda con los resultados obtenidos por López-Vázquez et al. (2015) en la región Mazahua del Estado de México, donde encontraron que el cambio de uso de suelo se da principalmente por el crecimiento de la población y la demanda de diferentes recursos naturales que requiere. Por el contrario, Ramos-Reyes et al. (2019) en un estudio realizado en Huimanguillo, Tabasco, encontraron que durante el periodo 2000-2010 hubo ganancia en los humedales y en la vegetación arbórea y uso urbano, pero existió una disminución de las áreas agropecuarias, manteniendo esta tendencia a la proyección realizada para el año 2030. Esto debido al establecimiento de grandes extensiones de plantaciones forestales comerciales en la región.

La dinámica del cambio de uso del suelo en la subcuenca Río Copalita se debe principalmente a los sistemas de producción agrícola y pecuario, sistemas de subsistencia que la población utiliza para autoconsumo, además del aprovechamiento de madera para la construcción de cercos y viviendas, los cuales compiten con la frontera forestal. En los últimos años, la presencia de plagas, enfermedades e incendios forestales, han favorecido el deterioro de los diferentes ecosistemas forestales. Actualmente existe una mayor conciencia en los ejidos, comunidades y pequeños propietarios, en cuanto a proteger y conservar los recursos naturales, como los bosques, el agua y el suelo, debido a los diferentes bienes y servicios ecosistémicos que ofrecen a la sociedad. Así mismo, en la parte media de la subcuenca se ha implementado un sistema agroforestal (producción de café bajo sombra) como una alternativa de recuperación de las áreas, el cual ha permitido mantener la cobertura vegetal, pero con una diversidad mucho menor y una estructura menos compleja; además, se ha logrado la generación de empleos para la población local (García-Alvarado et al. 2017).

En los últimos años, el ecoturismo ha sido 
impulsado en la subcuenca Río Copalita, logrando con ello un mayor beneficio para los dueños de las tierras y bosques por la derrama económica que genera, así como también, el establecimiento de plantaciones de árboles de navidad y sistemas agroforestales principalmente en la zona de Suchixtepec, mientras que en la parte media de la subcuenca domina la producción de café bajo sombra. Lo anterior ha propiciado un mejor manejo mediante la implementación de diferentes prácticas que permiten proteger el suelo, el agua y la biodiversidad (Mansourian et al. 2020). Por otra parte, VelascoMurguía et al. (2013) evaluaron 11 comunidades chinantecas de Oaxaca, encontrando que aquellas que contaban con asesoría técnica y realizaban investigaciones, favorecieron la recuperación de la cobertura arbolada, ya que recibían apoyos para proyectos de conservación y restauración de los ecosistemas. Esta situación es similar para las comunidades de la subcuenca Río Copalita, las cuales han recibido apoyo de diferentes proyectos en los últimos años, los cuales han permitido una mayor restauración y conservación de áreas prioritarias (Mansourian et al. 2020).

\section{Estimación del cambio de uso de suelo}

La tasa de cambio de uso de suelo y cobertura varió de -0.05 a $1.12 \%$. Los valores de $B Q P, B P Q$, BMM, SAB, SMC, SMS son similares a los reportados a nivel nacional por Mas et al. (2009), quienes encontraron valores de: $-0.25 \%$ para bosques, $-0.76 \%$ para selva y $-0.33 \%$ para matorral. También son semejantes a los resultados que la FAO (2015) reporta para México, con una tasa de cambio de vegetación primaria de $-0.6 \%$ para el periodo $2000-2010$, y de $-0.7 \%$ durante 1990-2015. Con base en lo anterior, Madrid et al. (2009) mencionan que es importante conocer las causas por las cuales se dan esos cambios, ya que más del $60 \%$ de los bosques están en ejidos y comunidades. La tenencia de la tierra ha sido clave en la subcuenca Río Copalita, ya que las primeras reforestaciones las establecieron pequeños propietarios del municipio de San Sebastián Río Hondo y San Miguel Suchixtepec, las cuales sirvieron como áreas demostrativas y experimentales para que otros propietarios replicaran la experiencia en sus tierras, de donde actualmente están obteniendo múltiples beneficios.

Las plantaciones y reforestaciones establecidas con especies nativas han permitido la recuperación de aquellas áreas que anteriormente se dedicaban a la agricultura y al pastoreo, lo cual ha favorecido a la menor escorrentía, mayor infiltración y recarga de los acuíferos, lo que se puede ver en la recuperación de manantiales, así como en la fauna silvestre, entre otros servicios ambientales. Para la parte media de la subcuenca, se ha favorecido el establecimiento del sistema agroforestal principalmente de café bajo sombra en áreas que fueron abandonadas por la agricultura. Esto ha permitido a los propietarios tener un ingreso económico, pero también ha permitido la recuperación de la vegetación del lugar, siendo una alternativa viable para la región, siempre y cuando no se desmonten las áreas con vegetación natural para establecer otros cultivos.

\section{CONCLUSIONES}

Los cambios de uso de suelo y cobertura de la subcuenca Río Copalita han sido dinámicos. La tasa de cambio positivo del bosque de pino, se debe a las reforestaciones y plantaciones a pequeña escala que se han establecido. Pero la pérdida de cobertura en los demás usos se debió a la apertura de nuevas áreas para la agricultura y pastizal. En las últimas dos décadas, debido a la implementación de programas de gobierno y asistencia técnica en los ejidos, comunidades y pequeños propietarios, así como al buen manejo y aprovechamiento forestal que se práctica en la parte alta y media de la subcuenca, se ha provocado la recuperación de las áreas abandonadas y/o degradadas. El uso de imágenes satelitales de alta resolución y del sistema de información geográfica (SIG) mejoró la precisión e interpretación de los cambios causados por las diferentes actividades antropogénicas que se desarrollaron en el periodo evaluado. 


\section{AGRADECIMIENTOS}

Al CONACYT por la beca de doctorado (CVU:1000961) otorgada al primer autor. Así mismo los autores agradecen a revisores anónimos y al editor asociado por sus acertados comentarios y observaciones para mejorar el manuscrito.

\section{LITERATURA CITADA}

Brauman KA, Freyberg DL, Daily GC (2014) Impacts of land-use change on groundwater supply: Ecosystem services assessment in Kona, Hawaii. Journal of Water Resources Planning and Management 141. DOI: 10.1061/(ASCE)WR.1943-5452.0000495.

Camacho-Sanabria JM, Juan-Pérez JL, Pineda-Jaimes NB, Cadena-Vargas EG, Bravo-Peña LC, Sánchez-López M (2015) Cambios de cobertura/uso del suelo en una porción de la Zona de Transición Mexicana de Montaña. Madera y Bosques 21: 93: 112.

Chuvieco E (2002) Teledetección ambiental: la observación de la Tierra desde el espacio. Editorial Ariel Ciencia. Barcelona, España. 586p.

CONAFOR (2016) Estudio de cuenca de abasto para el desarrollo industrial forestal maderable de la región Copalita, Oaxaca. Comisión Nacional Forestal. Colegio de Profesionales Forestales de Oaxaca, A. C. 309p. https://www.gob.mx/cms/uploads/attachment/file/254061/Estudio_de_cuenca_de_abasto_-_Copalita_Oaxa ca.pdf. Fecha de consulta: 15 diciembre de 2020.

CONAFOR (2020) Estimación de la tasa de deforestación bruta mediante el enfoque de muestreo. Comisión Nacional Forestal. 64p. https://www.gob.mx/cms/uploads/attachment/file/585544/6._Oswaldo_Carrillo_CONA FOR_Tasa_de_Deforestacion_16_Oct_2020.pdf. Fecha de consulta: 25 de noviembre de 2020.

Ellis EA, Romero-Montero JA, Hernández-Gómez IU, Anta-Fonseca S, López-Paniagua JE (2016) Determinantes de deforestación en el estado de Oaxaca. Agencia de los Estados Unidos para el Desarrollo Internacional (USAID), The Nature Conservancy (TNC), Alianza México REDD+. México 115p.

Escandón-Calderón J, Ordoñez-Díaz JAB, Nieto-de-Pascual-Pola MC del C, Ordoñez-Díaz M de J (2018) Cambio en la cobertura vegetal y uso del suelo del 2000 al 2009 en Morelos, México. Revista Mexicana de Ciencias Forestales 9: 27-51.

FAO (2015) Evaluación de los recursos forestales mundiales 2015, compendio de datos. Roma, Italia. 253p. http://www.fao.org/3/a-i4808s.pdf. Fecha de consulta: 20 de octubre de 2020.

García E (2004) Modificaciones al Sistema de Clasificación Climática de Köppen. Serie Libros, Núm. 6. Instituto de Geografía, Universidad Nacional Autónoma de México. México. 97p.

García-Alvarado ME, Diaz-Zorrillo GO, Castañeda-Hidalgo E, Lozano-Trejo S, Pérez-León MI (2017) Caracterización del agroecosistema de café bajo sombra en la cuenca del río Copalita. Revista Mexicana de Agronegocios 40: 635-648.

Gonzaga-Aguilar C (2015) Aplicación de índices de vegetación derivados de imágenes satelitales para análisis de coberturas vegetales en la provincia de Loja, Ecuador. CEDAMAZ 5: 30-41.

Ihl TJ, Bautista-Zúñiga F (2019) Estado actual de la cobertura y uso del suelo. En: CONABIO (ed). La biodiversidad en Michoacán. Estudio de Estado 2. CONABIO. México. pp: 61-65.

INEGI (2004) Síntesis de la información geográfica del estado de Oaxaca. Instituto Nacional de Estadística, Geografía e Informática. Primera edición. Aguascalientes, México. 180p. 
INEGI (2014a) Conjunto de datos vectoriales edafológico. Escala 1:250 000 Serie II. Continuo Nacional. Edición: 2. Instituto Nacional de Estadística y Geografía. Aguascalientes, Aguascalientes, México. https://www.inegi. org.mx/temas/edafologia/\#Descargas. Fecha de consulta: 20 de octubre de 2020.

INEGI (2014b) Guía para la interpretación de cartografía: uso del suelo y vegetación. Escala 1:250 000: Serie V. Instituto Nacional de Estadística y Geografía. Aguascalientes, México. 132p.

INEGI (2017) Conjunto de datos vectoriales de Uso de Suelo y Vegetación. Escala 1:250 000. Serie VI. Escala: 1:250 000. edición: 1. Instituto Nacional de Estadística y Geografía. Aguascalientes, México. https://www.inegi.org.mx/temas/usosuelo/\#Descargas. Fecha de consulta: 21 de octubre de 2020.

INEGI (2020) Censo de Población y Vivienda 2020. Instituto Nacional de Estadística y Geografía. Aguascalientes, México. https://www.inegi.org.mx/programas/ccpv/2020/. Fecha de consulta: 05 de febrero de 2021.

IUSS-WRB-FAO (2015) Base referencial mundial del recurso suelo 2014, Sistema internacional de clasificación de suelos para la nomenclatura de suelos y la creación de leyendas de mapas de suelos. Unión Internacional de las Ciencias del Suelo-Centro Internacional de Información y Referencia de Suelos-Organización de las Naciones Unidas para la Alimentación y Agricultura. Actualización 2015. Informes sobre recursos mundiales de suelos 106. Roma, Italia. 218p.

Leija-Loredo EG, Reyes-Hernández H, Reyes-Pérez O, Flores-Flores JL, Sahagún-Sánchez FJ (2016) Cambios en la cubierta vegetal, usos de la tierra y escenarios futuros en la región costera del estado de Oaxaca, México. Madera y Bosques 22: 125-140.

López-Vázquez VH, Valderas-Plata MA, Chávez-Mejía MC, Juan-Pérez JI, Gutiérrez-Cedillo JG (2015) Cambio de uso de suelo e implicaciones socioeconómicas en un área mazahua del altiplano mexicano. Ciencia Ergo-Sum 22: 136-144.

Madrid L, Núñez JM, Quiroz G, Rodríguez Y (2009) La propiedad social forestal en México. Investigación Ambiental 1: 179-196.

Mansourian S, González-Mora ID, Palmas-Tenorio MA, Spota-Diericx G, Vallauri D (2020) Lessons learnt from 15 years of integrated watershed management and forest restoration: the Copalita-Zimatán-Huatulco landscape in Mexico. Paris: WWF France, WWF report, field series, Experiences in Forest Landscape Restoration. Paris, France. 44p.

Mas JF, Velázquez A, Couturier S (2009) La evaluación de los cambios de cobertura/uso del suelo en la República Mexicana. Investigación Ambiental 1: 23-39.

Mejía-Domínguez NR, Meave JA, Ruiz-Jiménez CA (2004) Análisis estructural de un bosque mesófilo de montaña en el extremo oriental de la Sierra Madre del Sur (Oaxaca), México. Botanical Sciences 74: 13-29.

Moreno-Unda AA, Aguilar RM, Avalos LJA (2019) El Programa Nacional de Desmontes de México. En: Aguilar RM, Reyes HH, Reyes PO (ed). La Historia Ambiental en México: Estudios de Caso. Universidad Autónoma de San Luis Potosí. Facultad de Ciencias Sociales y Humanidades. México. pp: 117-127.

Nené-Preciado AJ, González-Sansón G, Mendoza ME, Silva-Bátiz FA (2017) Cambio de cobertura y uso de suelo en cuencas tropicales costeras del Pacifico central mexicano. Investigaciones geográficas, Boletín del Instituto de Geografía UNAM 94: 64-81.

Palacio-Prieto JL, Sánchez-Salazar MT, Casado-Izquierdo JM, Propin-Frejomil E, Delgado-Campos J, VelázquezMontes A, Chias-Becerril L, Ortiz-Álvarez MI, González-Sánchez J, Negrete-Fernández G, Gabriel-Morales J, Márquez Huitz R (2004) Indicadores para la Caracterización y Ordenamiento del Territorio. Instituto 
Nacional de Ecología-SEMARNAT, Instituto de Geografía de la UNAM y Secretaría de Desarrollo Social. México. 161p.

Pauleit S, Ennos R, Golding Y (2005) Modeling the environmental impacts of urban land use and land cover change: a study in Merseyside, UK. Landscape and Urban Planning 71: 295-310.

Pennington TD, Sarukhán J (2005) Árboles tropicales de México: manual para la identificación de las principales especies. Texto Científico Universitario. Fondo de la Cultura Económica. Universidad Nacional Autónoma de México. México. 523p.

Perry JP Jr (1991) The pines of Mexico and Central America. Timber Press. Portland, Oregon. 231p.

Pontius RG, Shusas E, McEachern M (2004) Detecting important categorical land changes while accounting for persistence, Agriculture. Ecosystem and Environment 101: 251-268.

QGIS Development Team (2019) QGIS Geographic Information System. Open Source Geospatial Foundation Project. https://qgis.org. Fecha de consulta: 12 de octubre de 2020.

Ramos-Reyes R, Palomeque de la Cruz MA, Núñez JC, Sánchez-Hernández R (2019) Análisis geomático espacial del cambio de uso del suelo en Huimanguillo, Tabasco (2000-2010-2030). Revista Mexicana de Ciencias Forestales 10: 118-139.

Sandoval JA, Tiburan CL Jr (2019) Identification of potential artificial groundwater recharge sites in mount makiling forest reserve, Philippines using GIS and analytical hierarchy. Applied Geography 105: 73-85.

Salas-Morales SH, Schibli L, Nava-Zafra A. y Saynes-Vásquez A (2007) Flora de la costa de Oaxaca, México (2): lista florística comentada del Parque Nacional Huatulco. Boletín de la Sociedad Botánica de México 81: 101-130.

SASPlanet (2018) SASPlanet Software. Versión 181221. SASPlanet Development Team. www.sasgis.org. Fecha de consulta: 20 de noviembre de 2020.

SEMARNAP (2000) Conservación y manejo comunitario de los recursos forestales en Oaxaca. México. Secretaria de Medio Ambiente Recursos Naturales y Pesca. Tlalpan, México. 212p.

Velasco-Murguía A, Duran-Medina E, Rivera R, Barton-Bray D (2013) Cambios en la cobertura arbolada de comunidades indígenas con y sin iniciativas de conservación, en Oaxaca, México. Investigaciones Geográficas, Boletín del Instituto de Geografía UNAM 83: 55-73. 